\title{
Medical Imaging
}

\section{Automatic planning of atrial fibrillation ablation lines using landmark-constrained nonrigid registration}

Martin Koch

Alexander Brost

Felix Bourier

Joachim Hornegger

Norbert Strobel 


\title{
Automatic planning of atrial fibrillation ablation lines using landmark-constrained nonrigid registration
}

\author{
Martin Koch, ${ }^{a, *}$ Alexander Brost, ${ }^{b}$ Felix Bourier, ${ }^{c}$ Joachim Hornegger, ${ }^{\text {a,d }}$ and Norbert Strobel ${ }^{\mathrm{e}}$ \\ ${ }^{a} F$ riedrich-Alexander-Universität Erlangen-Nürnberg, Pattern Recognition Lab, Martensstr. 3, Erlangen 91058, Germany \\ bStanford University, Department of Radiology, Stanford, California 94305 \\ 'Krankenhaus Barmherzige Brüder, Klinik für Herzrhythmusstörungen, Regensburg 93049, Germany \\ dErlangen Graduate School in Advanced Optical Technologies, Erlangen 91052, Germany \\ ésiemens AG, Forchheim 91301, Germany
}

\begin{abstract}
Catheter ablation is a common treatment option for drug-refractory atrial fibrillation. In many cases, pulmonary vein isolation is the treatment of choice. With current fluoro overlay methods or electroanatomic mapping systems, it is possible to visualize three-dimensional (3-D) anatomy as well as target ablation lines to provide additional context information. Today, however, these lines need to be set manually before the procedure by the physician, which may interrupt the clinical workflow. As a solution, we present an automatic approach for the planning of ablation target lines. Our method works on surface models extracted from 3-D images. To propose suitable ablation lines, a reference model annotated with reference ablation lines is nonrigidly registered to the model segmented from a new patient's 3-D data. After registration, the reference plan is transferred from the reference anatomy to the individual patient anatomy. Due to the high anatomical variations observed in clinical practice, additional landmark constraints are employed in the registration process to increase the robustness of our approach. We evaluated our method on 43 clinical datasets by benchmarking it against professionally planned ablation lines and achieved an average error over all datasets of $2.7 \pm 2.0 \mathrm{~mm}$. A qualitative evaluation of the ablation planning lines matched clinical expectations. ๑ 2014 Society of Photo-Optical Instrumentation Engineers (SPIE) [DOI: 10.1117/1.JMI.1.1.015002]
\end{abstract}

Keywords: treatment planning; image-guided procedures; landmark-constrained registration; electrophysiology; atrial fibrillation. Paper 13010RR received Oct. 23, 2013; revised manuscript received Apr. 11, 2014; accepted for publication Apr. 28, 2014; published online May 22, 2014.

\section{Introduction}

A common treatment option for drug-refractory atrial fibrillation (AFib) is minimally invasive catheter ablation. The recommended approach for AFib ablation is to block the electrical pathways from the pulmonary veins (PVs) into the left atrium (LA). This may be achieved by encircling both PV ostia with a single-wide circumferential line. ${ }^{1}$ The reported mid-term success rate for paroxysmal AFib is over $70 \%,{ }^{2}$ but results vary widely depending on the definition of success, ablation technique, experience, follow-up, and use of antiarrhythmic therapy. ${ }^{1}$

Pulmonary vein isolation (PVI) can be achieved by placing radiofrequency $(\mathrm{RF})$ lesions in the vicinity of the PVs. Two common strategies to perform PVI are segmental ablation and wide area circumferential ablation (WACA) around the ipsilateral PVs. ${ }^{1}$ The influence of the isolation area around the PVI on the procedure outcome has been investigated by Arentz et al. ${ }^{3}$ Their findings show that the ipsilateral ablation strategy is advantageous. ${ }^{3}$

In current clinical routine, a three-dimensional (3-D) image of the heart is acquired either before the case or at the beginning of the procedure. This 3-D image can either be imported into mapping systems such as CARTO 3 (Biosense Webster, Diamond Bar, California), EnSite Velocity (St. Jude Medical, St. Paul, Minnesota) or be superimposed directly on fluoroscopy to guide ablation catheters and mark ablation sites, for example

*Address all correspondence to: Martin Koch, E-mail: Martin.Koch@cs.fau.de by using syngo InSpace EP (Siemens Healthcare, Forchheim, Germany). If 3-D anatomical information is available, it can also be annotated with preferable ablation regions to obtain additional context information. ${ }^{4,5}$ Annotating ablation target sites on this 3-D dataset clearly identifies areas of interest during the ablation procedure. However, if preprocedural planning is to be performed during today's clinical workflow, it has to be carried out manually before the procedure starts. Since this can be difficult in a clinical environment due to time and workflow constraints, methods for automatic planning are desirable. A first automatic planning system for PVI ablation was proposed by Keustermans et al. ${ }^{6}$ In this approach, a local appearance model and a local shape model are trained for each ablation point. The local appearance model is based on intensity patterns in the image and trained on rotational angiography datasets. Since it is intensity based, applying this method to datasets acquired with different imaging protocols or imaging modalities, e.g., magnetic resonance imaging (MRI), requires additional adjustments. Keustermans et al.'s approach was evaluated on 3-D rotational angiography datasets for single PV isolation and ipsilateral PV isolation, respectively. They report an average distance error from ground truth for both ablation strategies of $3.8 \pm$ $2.9 \mathrm{~mm}$ for their automatic approach.

In this article, we present a new method for automatic lesion planning. It does not depend on intensity values. Instead, it involves a common reference model of the LA represented as a surface mesh. ${ }^{7}$ This model is annotated with reference ablation 
lines and then registered to the LA of each new patient represented as a template model. After registration, the planning lines are transferred from the reference model to the template model. To obtain robust results in the presence of significant anatomic variations among left atria from different patients, a landmarkconstrained nonrigid registration approach that treats landmarks as Dirichlet boundary conditions is used. ${ }^{8}$ Unlike other methods that integrate landmarks via Lagrange multipliers, ${ }^{9}$ this registration method does not increase, but reduces the numerical complexity by introducing additional landmarks as stated by Daum. ${ }^{8}$ The goal of this article is to investigate whether automatic annotation of a 3-D model yields reliable results. The use of reference ablation lines for PVI procedures offers interesting possibilities when applied across multiple physicians or labs. First, they convey expert knowledge to less experienced users. Second, reference ablation lines allow for easier comparison of different PVI strategies.

This article is structured as follows: We first describe our method for automatic planning of ablation lines via nonrigid landmark-constrained registration. Afterward, we evaluate the feasibility of our method on clinical datasets and present results. Finally, we discuss our findings and draw conclusions from our work.

\section{Materials and Methods}

We used contrast-enhanced 3-D MRI datasets to generate surface models of the LA for planning. The T1-weighted images were acquired on a 1.5 T Siemens MAGNETOM Avanto scanner (Siemens Healthcare, Erlangen, Germany) using a spoiled 3 -D gradient echo sequence. The average resolution of the reconstructed 3 -D volumes was $256 \times 256 \times 81$ voxel, with an in-plane pixel spacing of $1.14 \times 1.14 \mathrm{~mm}$, and a slice thickness of $1.57 \mathrm{~mm}$. A semiautomatic segmentation software (syngo InSpace EP, Siemens Healthcare, Forchheim, Germany) was used to extract the LA surface model from the 3-D volume. The segmentation process was manually initialized by selecting a voxel in the 3-D volume, corresponding to an area inside the LA. Based on this seedpoint, the complete LA was segmented automatically. This semiautomatic segmentation step was part of the routine clinical workflow at this site. The surface model is represented as a triangulated mesh structure. The models are constrained to cover the LA as well as a certain extent of the attached PVs, from the ostium to about $2 \mathrm{~cm}$ distal of the first branch. In our planning framework, the mesh is seen as a point set $\boldsymbol{m}$ comprising $N_{p}$ points $\boldsymbol{x}_{i} \in \mathbb{R}^{3}$

$\boldsymbol{m}=\left\{\boldsymbol{x}_{1}, \ldots, \boldsymbol{x}_{N_{p}}\right\}$.

Our data pool is composed of $N_{t}$ template meshes $\boldsymbol{m}_{t}$ with $t=1, \ldots, N_{t}$ and a reference mesh $\boldsymbol{m}_{\text {ref }}$. The spatial orientations of the meshes are determined by the digital imaging and communications in medicine (DICOM) coordinate systems of the associated MRI volume datasets. Before processing, all meshes were zero centered. The reference mesh is a principal component analysis-based mean shape model of the LA. ${ }^{7,10}$ It was constructed from a representative subset of 10 meshes picked from the available template meshes. The datasets for computing the mean shape were selected by a visual inspection. We chose LA models that were characterized by a clear separation of PVs, antrum, and left atrial appendage. As we rely on a nonrigid landmark-constrained registration approach for automatic planning, landmarks need to be established in the proximity of the PV ostia as discussed in the following section.

\subsection{Landmark Extraction}

We were interested in landmarks in close proximity to the PV ostia. Our current segmentation algorithm only provided a complete structure of the LA combining body and the attached PVs. Note, however, that segmentation algorithms have been proposed that not only extract the LA, but also detect PV ostia. $^{11,12}$ Zheng et al., for example, developed an algorithm for LA segmentation which implicitly models the PV osia. They reported an accuracy of 1.61 to $1.96 \mathrm{~mm}$ for PV and LA chamber segmentation, respectively. To take additional uncertainties into account, we placed six to eight points around each PV within $5 \mathrm{~mm}$ distally to where the PV meets the LA chamber.

To fully encircle each PV ostium, these 3-D point sets were interpolated using cubic splines. Finally, a fixed number of landmarks $\boldsymbol{b}_{i}$ were extracted automatically along the splines. Landmarks $\boldsymbol{b}=\left\{\boldsymbol{b}_{i}\right\}_{1}^{B}$ were represented as points with $\boldsymbol{b}_{i} \in \mathbb{R}^{3}$. On each PV ostium, four landmarks were selected, located at the top, bottom, anterior and posterior sides, respectively, as defined by the underlying DICOM coordinate system of the dataset. Wittkampf et al. ${ }^{13}$ found that the overall shape of the PV ostium is oval, with the short axis in the anterior/posterior directions. This is why the spline curves were cut with two planes passing through the center of the corresponding PV ostium, where the first plane is parallel to the $(x, y)$-plane (axial plane), and the second plane was constructed to be parallel to the $(x, z)$-plane (coronal plane). Since left atria have four PVs, this results in a total of $B=16$ landmarks. An example of a rendered LA model with annotated landmarks around four PV ostia is shown in Fig. 1. A schematic drawing of the landmark arrangement for a single PV ostium can be seen in Fig. 2. The landmark extraction was performed once for the reference model $\boldsymbol{m}_{\text {ref }}$ and subsequently for every template mesh to be processed.

\subsection{Nonrigid Landmark-Constrained Registration}

Registration was performed using the nonrigid landmarkconstrained registration approach proposed by Daum. ${ }^{8}$ As the proposed algorithm works on 3-D voxel datasets, we converted the triangle mesh $\boldsymbol{m}$ into a corresponding binary volume.

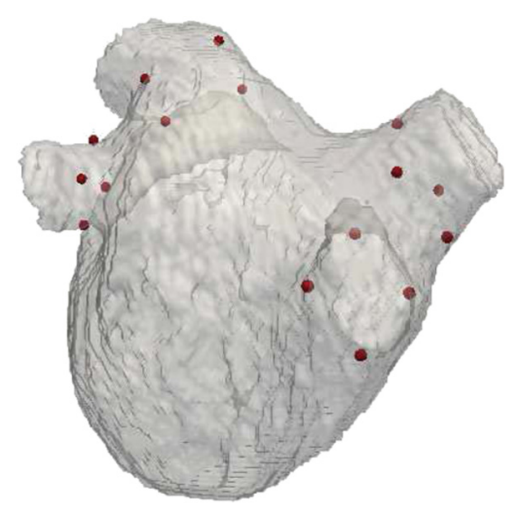

Fig. 1 Model of the left atrium (LA) with landmarks $\boldsymbol{b}_{i}$ placed around each pulmonary vein (PV) ostia. The LA model is shown in a lateral orientation with a direct view into the right inferior PV. 


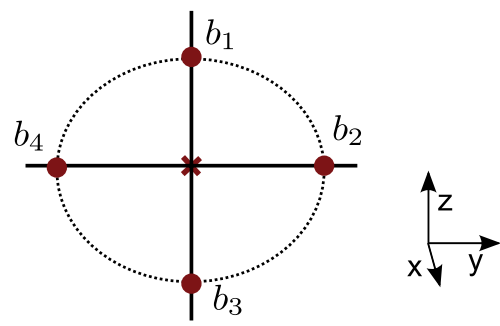

Fig. 2 Schematic drawing of the landmark arrangement for a single PV ostium. The dotted line represents the PV ostium. The two solid lines represent cutting planes in three-dimensional crossing through the center of the PV ostium, depicted by the bold cross. Red dots at the crossings of solid and dotted lines represent the respective landmarks $\boldsymbol{b}_{i}$.

In the following section, $R$ and $T$ refer to the binary volume representation of the corresponding datasets. The general nonrigid registration problem can be described as finding a mapping $\boldsymbol{u}: \mathbb{R}^{3} \rightarrow \mathbb{R}^{3}$ between a reference image $R$ and a template image $T$, such that the transformed template $T_{\boldsymbol{u}}(\boldsymbol{x})=T[\boldsymbol{x}-\boldsymbol{u}(\boldsymbol{x})]$ is as similar to $R$ as possible. Mathematically formulated, we are looking for the optimal mapping $\boldsymbol{u}^{*}$ by minimizing the following energy function:

$\boldsymbol{u}^{*}:=\arg \min _{\boldsymbol{u}} \mathcal{D}\left(R, T_{\boldsymbol{u}}\right)+\alpha \mathcal{R}(\boldsymbol{u})$,

where $\mathcal{D}(\cdot)$ is the image matching term, $\mathcal{R}(\cdot)$ is the regularizer enforcing smoothness on $\boldsymbol{u}$, and $\alpha \in \mathbb{R}$ is a weighting factor. We use the sum of the squared differences as the distance measure $\mathcal{D}:{ }^{14}$

$\mathcal{D}_{\mathrm{SSD}}\left(R, T_{\boldsymbol{u}}\right):=\frac{1}{|\Omega|} \int_{\Omega}\left[R(\boldsymbol{x})-T_{\boldsymbol{u}}(\boldsymbol{x})\right]^{2} \mathrm{~d} \boldsymbol{x}$.

$\Omega \in \mathbb{R}^{3}$ represents the computational domain of the registration, and $|\Omega|$ represents the corresponding area. To enforce a smooth deformation field, we make use of the curvature regularizer: ${ }^{15}$

$\mathcal{R}_{\text {Curv }}(\boldsymbol{u}):=\frac{1}{|\Omega|} \int_{\Omega}[\Delta \boldsymbol{u}(\boldsymbol{x})]^{2} \mathrm{~d} \boldsymbol{x}$.

To solve Eq. (2), the Euler-Lagrange equation is calculated with respect to the unknown deformation $\boldsymbol{u}$. In our case, this results in the following partial differential equation

$$
\left[R(\boldsymbol{x})-T_{\boldsymbol{u}}(\boldsymbol{x})\right] \nabla T_{\boldsymbol{u}}(\boldsymbol{x})+\alpha\left(\Delta^{2} \boldsymbol{u}\right)(\boldsymbol{x})=0 \quad \forall \boldsymbol{x} \in \Omega,
$$

where $\nabla$ is the gradient operator and $\Delta$ is the Laplace operator. To actually compute a solution, the problem needs to be discretized. The registration domain $\Omega$ is discretized on a regular grid with homogeneous spacing $h$. The differential operator $\Delta^{2}$ can be described by a matrix $\mathbf{A}^{8}$ The deformation field $\boldsymbol{u}$ is discretized as a vector $\mathbf{u}_{i}=\boldsymbol{u}\left(\boldsymbol{x}_{i}\right) \quad \forall \boldsymbol{x}_{i} \in \Omega$. For the optimization itself, a semi-implicit gradient descent scheme is used

$$
(\mathbf{I}+\tau \alpha \mathbf{A}) \mathbf{u}^{(t+1)}=\mathbf{u}^{(\mathbf{t})}-\tau\left[R(\boldsymbol{x})-T_{\mathbf{u}^{(t)}}(\boldsymbol{x})\right] \cdot \nabla T_{\mathbf{u}^{(t)}}(\boldsymbol{x}),
$$

with iteration index $t$ and step size $\tau$.
To integrate landmarks into the registration framework, the known correspondences between reference and template images are removed from the computational domain. Correspondences in our case are defined as point-to-point mappings between locations in the reference image $x_{R} \in \Omega_{R}$ and the template image $\boldsymbol{x}_{T} \in \Omega_{T}$. The set of points with known correspondence is defined as $\Omega_{c} \subset \Omega$. The mapping of corresponding points can be described by the function $c: \Omega_{c} \rightarrow \mathbb{R}^{3}$, hence

$\boldsymbol{x}_{T}=\boldsymbol{c}\left(\boldsymbol{x}_{R}\right) \quad \forall \boldsymbol{x}_{R} \in \Omega_{c}$.

Based on the known point correspondences, the transformation $\boldsymbol{u}\left(\boldsymbol{x}_{R}\right) \quad \forall \boldsymbol{x}_{R} \in \Omega_{c}$ is also known,

$\mathbf{u}_{i}=\boldsymbol{u}\left(\boldsymbol{x}_{i}\right)=\boldsymbol{x}_{i}-\boldsymbol{c}\left(\boldsymbol{x}_{i}\right) \quad \forall i: \boldsymbol{x}_{i} \in \Omega_{c}$.

This is used as the Dirichlet boundary condition in the optimization process. Thereby, the actual computational domain is reduced by every additional known correspondence. The remaining computational domain can be described as

$\tilde{\Omega}:=\Omega \backslash \Omega_{c}$.

In practical terms, these modifications are reflected in the system matrix $\mathbf{I}+\tau \alpha \mathbf{A}$ in Eq. (6). By initializing $\mathbf{u}_{i}^{(0)}$ with the known point correspondences, it will stay constant throughout the iteration. All remaining positions within the deformation field, including the area around the landmarks, are affected by the regularizer during the optimization. Thereby, a smooth deformation is produced. Due to the changes in the system matrix, the symmetry property is not generally fulfilled anymore. ${ }^{8}$ We used a stabilized bi-conjugate gradient method for solving the linear system. An example for a landmark initialization and the resulting deformation field after registration is shown in Fig. 3.

\subsection{Transfer of Planning Lines}

Planning lines $\boldsymbol{l}$ are represented as a set of points $\boldsymbol{l}=$ $\left\{\boldsymbol{x}_{1}, \ldots, \boldsymbol{x}_{P}\right\}$ with $\boldsymbol{x}_{i} \in \mathbb{R}^{3}$. To transfer the reference planning lines $\left\{\boldsymbol{l}_{\text {ref }, R}, \boldsymbol{l}_{\text {ref }, L}\right\}$ onto the template mesh $\boldsymbol{m}_{\mathbf{t}}, \boldsymbol{m}_{\mathbf{t}}$ is registered to the reference model $\boldsymbol{m}_{\text {ref }}$ using the landmark-constrained registration described above. After registration, the two mesh models are optimally aligned based on the optimization criterion stated in Eq. (2). The inverse deformation, sampled at discrete positions in the reference image space, is then applied to the reference planning lines. In a final step, the planning lines are readjusted on the surface of the template mesh by enforcing that the transfered planning points coincide with mesh vertices. In our current implementation, this is performed by repositioning the planning points to the closest mesh vertex. This operation is performed to ensure better visualization of the planning lines on the mesh surface. On the template model, the generated planning lines are labeled as $\hat{\boldsymbol{l}}_{t, R}$ and $\hat{\boldsymbol{l}}_{t, L}$, for right and left sided plannings, respectively.

\section{Experimental Results}

We evaluated our approach on 43 clinical datasets. According to the PV branching pattern classification scheme proposed by Kato et al., ${ }^{16} 39$ datasets contained left atria with a configuration of four distinct PV ostia, type A. This branching pattern also reflects the most common anatomic configuration. ${ }^{17}$ Three datasets had a common ostium on the left side, type C, and 


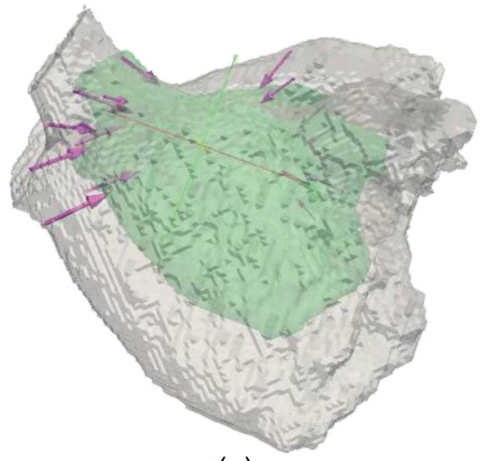

(a)

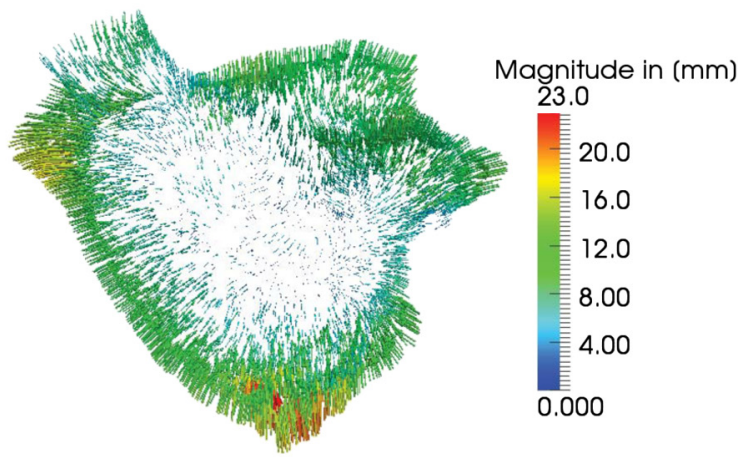

(b)

Fig. 3 (a) Two mesh models with landmark initialization visualized by purple vectors. Due to occlusion not all landmarks are visible. In the shown case, the template model is considerably larger compared with the inner reference model. (b) Deformation field after nonrigid landmark-constrained registration. The deformation vectors are color coded based on their magnitude. For visualization purposes only a subset of the deformation vectors is shown.

one dataset had an additional fifth middle PV on the right side, type D. An experienced clinician placed planning lines around the ipsilateral left and right PVs to provide ground truth. As a result, each template mesh $\boldsymbol{m}_{t}$ received two ground truth planning lines $\boldsymbol{l}_{t, R}$ and $\boldsymbol{l}_{t, L}$. They represent desirable ablation areas for right and left sided PVs, respectively. The planning lines are a subset of the corresponding template mesh, i.e., $\left\{\boldsymbol{l}_{t, R}, \boldsymbol{l}_{t, L}\right\} \subset$ $\boldsymbol{m}_{t}$. Our reference model with annotated ipsilateral planning lines and annotated PV ostia is shown in Fig. 4. The annotation of the reference model was also performed by an experienced clinician. This reflects the desired workflow: the physician provides annotations on a dedicated reference model based on his or her personal preferences once. This information is then used for automatic planning on new datasets.

The deviation of the automatically placed ablation planning lines $\hat{l}_{t, R}$ and $\hat{l}_{t, L}$ from the respective ground truth annotation was evaluated as follows. Each ablation line was interpolated with a cubic spline and equidistantly sampled at $P=30$ positions. For each sampled point, the minimal Euclidean distance to the corresponding ground truth planning lines $\boldsymbol{l}_{t, R}$ and $\boldsymbol{l}_{t, L}$ was calculated. The registration and automatic planning were performed on a regular desktop PC using a prototype implementation. Currently, our registration process takes about $35 \mathrm{~min}$ per dataset. Further code optimization and parallelization will lead to additional speed-up. In clinical workflow, the automatic planning could be initiated by technical or medical staff right after acquisition of the 3-D preprocedural images. Examples of

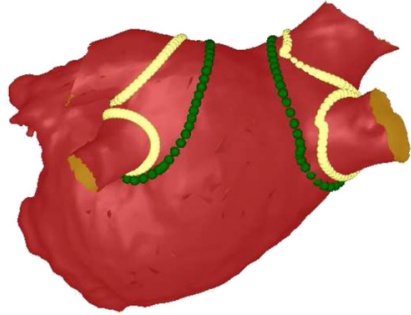

(a) Posterior view

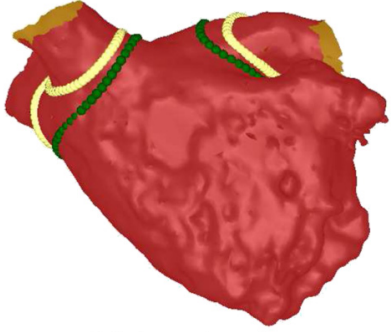

(b) Anterior view
Fig. 4 Reference model of the LA with annotated PV ostia (yellow), and ipsilateral ablation planning lines (green). The annotation of the PV ostia facilitates automatic PV landmark detection. The ipsilateral ablation lines are mapped from the reference model to the template model after nonrigid landmark-constrained registration. automatically planned ipsilateral ablation lines are shown in Fig. 5. The computed lines for ipsilateral ablation represent a suggestion that can always be manually adjusted by the clinician to ensure that the individual expectations are met.

The overall average distance of the automatically placed ipsilateral ablation lines to the corresponding ground truth annotations was $2.7 \pm 2.0 \mathrm{~mm}$. Taking a separate look at right and left planning lines, the average distance was $2.2 \pm 1.5$ and $3.1 \pm 2.4 \mathrm{~mm}$, respectively. The maximum distance of a singleplanning point to the reference planning line was 8.5 and $12.4 \mathrm{~mm}$ for right and left sided ablation lines, respectively. An evaluation of the error per dataset is shown in Fig. 6. For a more anatomically oriented evaluation, the planning lines were divided into eight equally spaced segments as depicted in Fig. 7. Based on this convention, we present the mean deviation of the planned ablation lines from the ground truth per line segment in Fig. 7. Line segments 1 and 2 are located on the roof of the LA, segments 3 and 4 are on the anterior side of the LA, segments 5 and 6 are below the inferior PV, and segments 7 and 8 are on the posterior side of the LA. The highest planning errors occur in segment 1 on the right side with an average of $3.5 \pm 1.9 \mathrm{~mm}$ and segment 2 on the left side with an average error of $3.6 \pm 2.6 \mathrm{~mm}$.

We also performed a qualitative evaluation of our results. An experienced clinician was asked to judge the usability of the automatically planned ablation lines, while being blinded to the ground truth annotations to ensure an unbiased evaluation.

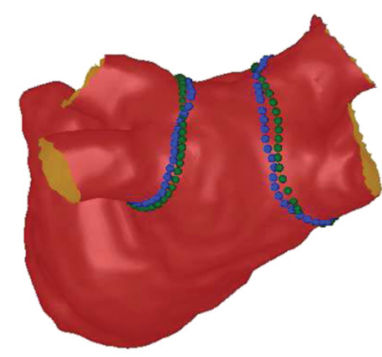

(a) Dataset 39

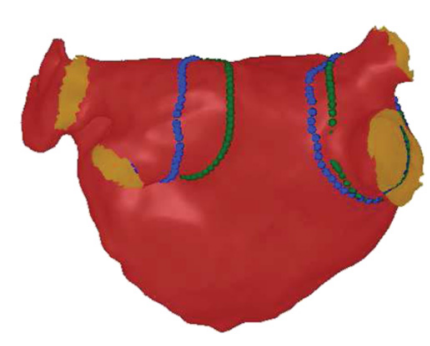

(b) Dataset 27
Fig. 5 Posterior view on datasets with automatically annotated ablation planning lines (blue) and ground truth annotation (green). (a) Dataset with low planning error. (b) Dataset with high error on the left PVs. 
(a) Error for right sided ablation lines

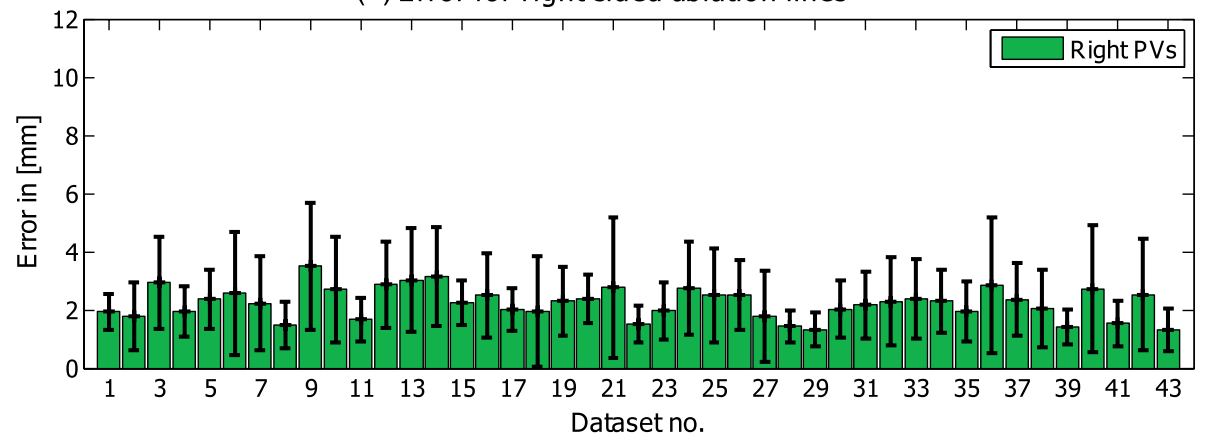

(b) Error for left sided ablation lines

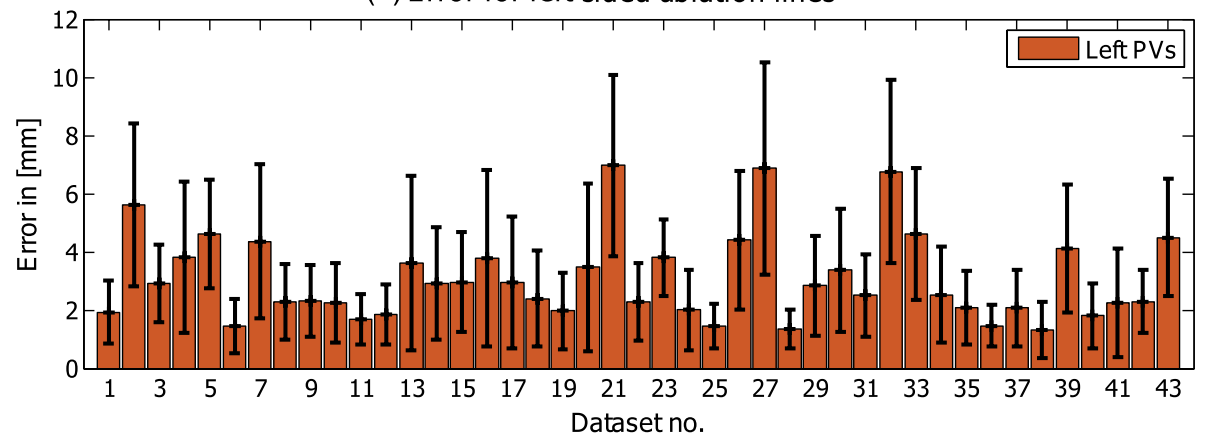

Fig. 6 Error of automatically planned ipsilateral ablation lines compared with ground truth annotations per dataset. The error is shown for each dataset separately as average \pm standard deviation. (a) Rightsided planning lines; (b) left-sided planning lines.

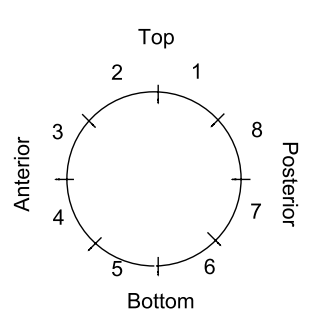

(a)

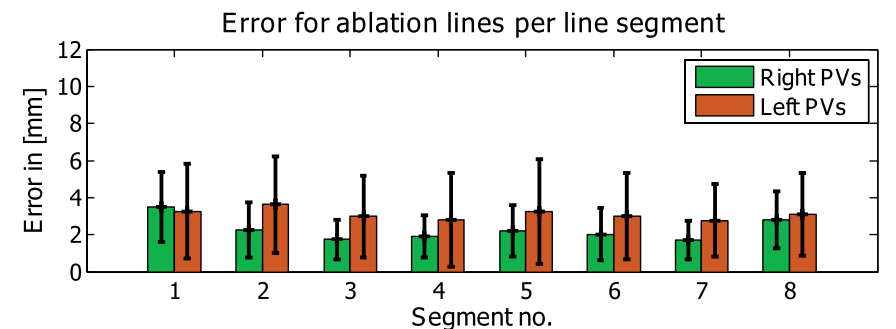

(b)

Fig. 7 (a) Labeling of clock view segments for spatial evaluation of planning accuracy. (b) Error of automatically planned ablation lines compared to ground truth annotations per segment. The error is shown as average \pm standard deviation over all datasets.

In particular, he was asked to classify them into four categories: (I) accepted without modifications, (II) accepted, but minor modifications suggested, (III) modifications needed, but initial suggestion helpful, and (IV) incorrect planning lines. The results are shown in Table 1 . In $86 \%$ of the datasets, the proposed planning was accepted without any modifications. In 14\%, minor

Table 1 Qualitative evaluation of automatically planned ablation lines. (I) planning lines accepted, (II) minor modifications suggested, (III) modifications needed, but initial suggestion helpful, and (IV) incorrect planning lines.

\begin{tabular}{lcccc} 
& \multicolumn{4}{c}{ Category } \\
\cline { 2 - 5 } Number of datasets & I & II & III & IV \\
\hline 43 & $86 \%$ & $14 \%$ & $0 \%$ & $0 \%$ \\
\hline
\end{tabular}

modifications were suggested. None of the datasets were classified in category (III) or (IV).

We also investigated the impact of the particular choice for the reference planning lines on the average error of our method. We recomputed the left (right) reference planning line as the mean of all available ground truth annotations at this side after nonrigid registration. Since this approach involves ground truth data, the results do not reflect actual algorithm performance. However, they can serve as the lower boundary on the planning error. Using this definition of reference annotations, a planning error of $2.4 \pm 2.0 \mathrm{~mm}$ was achieved. A visual comparison of the manually placed reference planning lines used during the evaluation and the mean planning line computed based on the ground truth annotations is shown in Fig. 8. We see that they are very close to each other. This explains why the planning error is very similar in both cases. It also shows that the clinician was very consistent when placing the ground truth planning annotations across the available mesh models. 


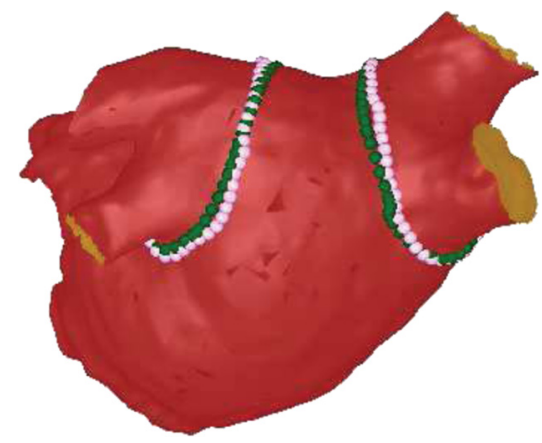

Fig. 8 Reference model of the LA with ground truth annotations (green) and mean ablation planning lines (pink). The mean planning lines were computed based on all ground truth annotations.

\section{Discussion}

Our landmark-constrained nonrigid registration algorithm ensures a one-to-one mapping of the landmarks, i.e., the landmark registration error equals zero. Taking into account (four) landmarks for each PV during the registration process, we were able to achieve a better match within the area of interest, i.e., the PV ostia, to automatically generate accurate planning results. The proposed method yields an overall average planning error of $2.7 \pm 2.0 \mathrm{~mm}$ for planning of ipsilateral PV ablation lines. Considering an ablation lesion size of about $5 \mathrm{~mm}$, our automatically proposed ablation lines appear sufficiently accurate. This is confirmed by the qualitative evaluation of the planning results, which met clinical expectations as shown in Table 1.

The highest planning errors occurred in datasets 21,27 , and 32 on the left sided PVs, respectively, as shown in Fig. 6. In those datasets, a common ostium was present. In Fig. 5, one LA with a common ostium on the left side is shown. Even though there was a higher planning error, compared to the ground truth annotations, the ablation planning lines were still classified as useful by the physician. This suggests that there seems to be a wider band of acceptable ablation lines in the case of a common ostium. Our reference model of the LA comprises four individual PV ostia, i.e., it does not have a common ostium. This reflects the most common configuration for left atria. ${ }^{17}$ To improve the planning accuracy for left atria with a common ostium, a separate reference model could be used.

We benchmarked our results against the state of the art as presented by Keustermans et al. ${ }^{6}$ Although our mean error of $2.7 \mathrm{~mm}$ implies a $29 \%$ improvement over the $3.8 \mathrm{~mm}$ obtained by Keusterman et al., both results appear sufficiently accurate from a clinical point of view, considering the typical ablation lesion size is around 5 to $7 \mathrm{~mm}$. Yet, there is an important difference between the two approaches. Although the method by Keusterman et al. was fine tuned for a particular 3-D imaging technique, 3-D rotational angiography, our approach is model based, i.e., it can be employed across different modalities as long as a segmentation (mesh) is available. Care has to be taken, however, to ensure that the spatial resolutions of the datasets involved are matched to each other. We also applied the method proposed in Ref. 18 to our current test dataset using nonrigid point cloud registration not involving any landmarks. This method only achieved an average planning error of $9.7 \pm 7.0 \mathrm{~mm}$ over all datasets, indicating that the use of landmark constraints offers significant improvements in registration performance.
For our evaluations, we used ground truth data provided by a single expert. To ensure that our results appear reasonable from a clinical point of view, we asked the expert to perform a qualitative evaluation of the automatic planning results. To avoid bias, no associated ground truth information was revealed to the expert. The expert found our results plausible and clinically acceptable. The success rate of an ablation procedure may not be dependent on the ablation planning only, but on many other factors as well. To evaluate the potential impact of automatically generated ablation plans on procedure outcome, an extensive medical study would be needed.

\section{Conclusions}

We presented a method for automatic planning of ipsilateral PV ablation lines. If implemented such that the physician can automatically and instantly obtain a suggestion for suitable ablation lines without any further effort, we expect that the use of planned ablation lines will become part of the clinical routine. Our proposed method involves landmarks placed at the PV ostia to generate individual planning lines based on landmark-constrained nonrigid registration. In our experiments, we achieved an average planning error of $2.7 \pm 2.0 \mathrm{~mm}$ on a diverse set of LA models. Our results meet clinical expectations, which were demonstrated in a qualitative evaluation by a physician. An extension to further applications involving physiological information, e.g., tissue characteristics, ${ }^{19}$ is feasible as well.

\section{Acknowledgments}

We thank Dr. K. Kurzidim (Krankenhaus Barmherzige Brüder, Regensburg, Germany) for the clinical data. This work was supported by the German Federal Ministry of Education and Research (BMBF) in the context of the initiative Spitzencluster Medical Valley-Europäische Metropolregion Nürnberg, project grant Nos. 13EX1012A and 13EX1012E, respectively. Additional funding was provided by Siemens AG, Healthcare Sector. J. Hornegger gratefully acknowledges funding of the Erlangen Graduate School in Advanced Optical Technologies (SAOT) by the German Research Foundation (DFG) in the framework of the German excellence initiative. The concepts and information presented in this article are based on research and are not commercially available.

\section{References}

1. H. Calkins et al., "2012 HRS/EHRA/ECAS expert consensus statement on catheter and surgical ablation of atrial fibrillation," Heart Rhythm 9(4), 632-696 (2012).

2. R. Tung, E. Buch, and K. Shivkumar, "Catheter ablation of atrial fibrillation," Circulation 126(2), 223-229 (2012).

3. T. Arentz et al., "Small or large isolation areas around the pulmonary veins for the treatment of atrial fibrillation?," Circulation 115(24), 3057-3063 (2007).

4. S. De Buck et al., "An augmented reality system for patient-specific guidance of cardiac catheter ablation procedures," IEEE Trans. Med. Imaging 24(11), 1512-1524 (2005).

5. F. Bourier et al., "Pulmonary vein isolation supported by MRI-derived 3D-augmented biplane fluoroscopy: a feasibility study and a quantitative analysis of the accuracy of the technique," J. Cardiovasc. Electrophysiol. 24(2), 113-120 (2013).

6. J. Keustermans et al., "Automated planning of ablation targets in atrial fibrillation treatment," Proc. SPIE 7962, 796207 (2011).

7. M. Koch et al., "Towards deformable shape modeling of the left atrium using non-rigid coherent point drift registration," in Bildverarbeitung für die Medizin, H.-P. Meinzer et al., Eds., Informatik aktuell, pp. 332-337, Springer, Berlin Heidelberg (2013). 
8. V. Daum, "Model-constrained non-rigid registration in medicine," $\mathrm{Ph} . \mathrm{D}$. Thesis, Friedrich-Alexander-Universität Erlangen-Nürnberg, Erlangen, Germany (2011).

9. B. Fischer and J. Modersitzki, "Combining landmark and intensity driven registrations," Proc. Appl. Math. Mech. 3(1), 32-35 (2003).

10. T. Heimann and H.-P. Meinzer, "Statistical shape models for $3 \mathrm{~d}$ medical image segmentation: a review," Med. Image Anal. 13(4), 543-563 (2009).

11. R. Karim, R. Mohiaddin, and D. Rueckert, "Left atrium pulmonary veins: segmentation and quantification for planning atrial fibrillation ablation," Proc. SPIE 7261, 72611 T (2009).

12. Y. Zheng et al., "Precise segmentation of the left atrium in C-arm CT volumes with applications to atrial fibrillation ablation," in 9th IEEE Int. Symposium on Biomedical Imaging, Barcelona, pp. 1421-1424, IEEE (2012).

13. F. H. Wittkampf et al., "Pulmonary vein ostium geometry," Circulation 107(1), 21-23 (2003).

14. J. Modersitzki, Numerical Methods for Image Registration (Numerical Mathematics and Scientific Computation), Oxford University Press, Oxford (2004).

15. B. Fischer and J. Modersitzki, "Curvature based image registration," J. Math. Imaging Vision 18(1), 81-85 (2003).
16. R. Kato et al., "Pulmonary vein anatomy in patients undergoing catheter ablation of atrial fibrillation: lessons learned by use of magnetic resonance imaging," Circulation 107(15), 2004-2010 (2003).

17. J. Kautzner, H. Micochova, and P. Peichl, "Anatomy of the left atrium and pulmonary veins-lessons learned from novel imaging techniques," European Cardiology 2(1), 89-90 (2006).

18. M. Koch et al., "Novel method for comparison of pre-planned ablation lines for treatment of atrial fibrillation using a common reference model," in 10th IEEE Int. Symposium on Biomedical Imaging, San Francisco, California, pp. 33-36, IEEE (2013).

19. R. S. Oakes et al., "Detection and quantification of left atrial structural remodeling with delayed-enhancement magnetic resonance imaging in patients with atrial fibrillation," Circulation 119(13), 1758-1767 (2009).

Martin Koch received his MS degree in computer science from the Friedrich-Alexander-Universität Erlangen-Nürnberg, Germany, in 2010. He is currently a PhD student at the Pattern Recognition Lab, Friedrich-Alexander-Universität Erlangen-Nürnberg, Erlangen, Germany. His research interests include image processing and analysis and medical imaging.

Biographies of the other authors are not available. 